\title{
Effect of synchronization mismatch on modulation instability in passive fiber-ring cavity
}

\author{
Stefano Negrini ${ }^{1}$, François Copie ${ }^{1}$, Saliya Coulibaly ${ }^{1}$, Matteo Conforti ${ }^{1}$, Alexandre Kudlinski ${ }^{1}$, and \\ Arnaud Mussot ${ }^{1}$ \\ ${ }^{1}$ Univ. Lille, CNRS, UMR 8523 - PhLAM - Physique des Lasers Atomes et Molécules, F-59000 Lille, France
}

Pulsed pumping configurations are becoming an interesting scheme for triggering optical frequency combs (OFC) in passive resonators due to their high peak power and to their low induced thermal loading [1]. A careful change of the repetition rate of these pumps compared to the repetition rate of the resonators is required to get an efficient OFC generation. Small mismatches, cumulated over many round trips, can lead to significant drifts leading to crucial modifications of the dynamics of the processes. A deep investigation of the impact of the synchronization mismatch on the complex building-up of OFC has never been reported yet. Here, for simplicity, we first restricted our study to the impact of the synchronization mismatch on modulation instability, because MI is the first and simplest nonlinear phenomenon appearing in a passive resonator and involved in the formation of OFCs. In its basic formulation, MI phenomenon depends only on the even terms of the dispersion [1]. However, in the real world experimentation, it has been reported that under synchronous pumping, and in the weak dispersion regime, the slope of the dispersion leads the system to become convectively unstable inducing a power asymmetry between the side-bands [2,3]. Here we worked in a strong dispersion regime where these effects can be neglected. The cavity is pumped with square shape pulses with $560 \mathrm{ps}$ duration, just above the MI threshold, (other parameters are listed in the figure's caption). We recorded the output spectra with an optical spectrum analyzer and the roundtrip to round-trip temporal evolution with a commercial time lens system (Picoluz). The evolution of the output spectra as a function of the synchronization mismatch is depicted in Fig. 1(a). For perfect synchronization $(\Delta \mathrm{T}=0)$, narrow MI sidebands are generated at $28.21 \mathrm{GHz}$ (see inset), in pretty good agreement with theoretical predictions $(40 \mathrm{GHz})$. Perfect synchronization operation is confirmed through the stable evolution of the pulse train roundtrips over round -trips (fig. 1(c)). By slightly changing the synchronization mismatch of only a few tens of fs, the position of the sideband is shifted by more than $12 \mathrm{GHz}$. For large mismatch values (Mismatch> $0.01 \mathrm{ps} / \mathrm{m}$ ), the positions of the sidebands are unchanged and they experience an important spectral broadening. This significant modification of the dynamics of the process is directly related to convective instabilities [2,3] associated with the synchronization mismatch as can be seen in Fig. 1(b) and (d). Theoretically, it means that, the first order dispersion term $\left(\beta_{1}\right)$ must be considered in the phase matching relation $[2,3]$.
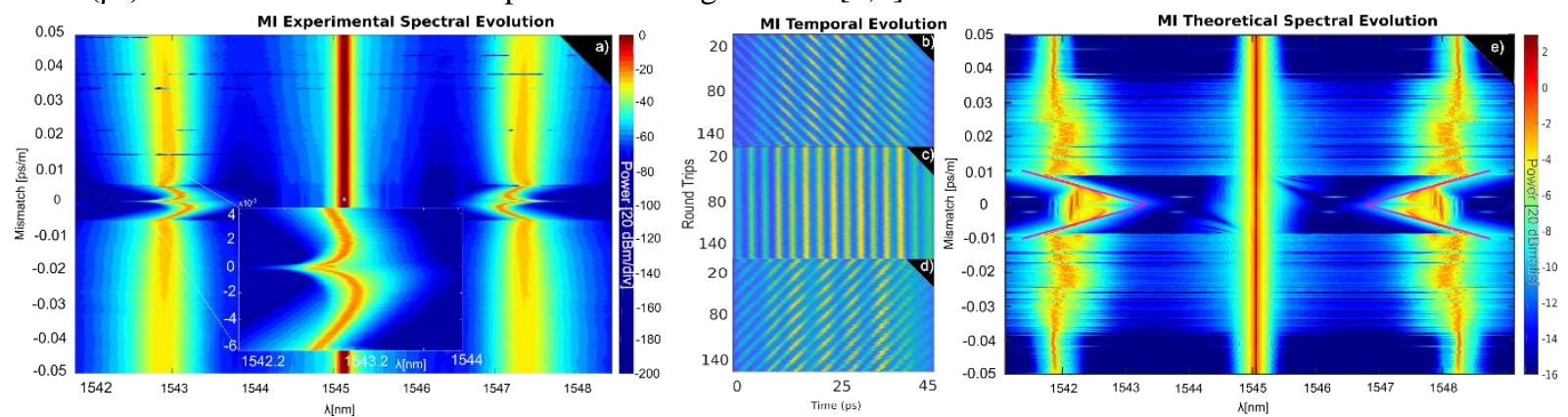

Fig. 1 : (a) 2D plot of the output MI spectra as a function of the synchronization mismatch. Parameters : Pulse width $=560 \mathrm{ps,}$ $\mathrm{L}=27.44 \mathrm{~m}, \gamma \approx 2.5 / \mathrm{W} / \mathrm{Km}, \beta_{2}=-3.8 \mathrm{ps}^{2} / \mathrm{km}, \mathrm{Pp}=10.3 \mathrm{~W}$. (b,c,d) Three typical temporal traces recorded with a time lens system at different mismatch values for $\Delta \mathrm{T}=0.024 \mathrm{ps}, 0 \mathrm{ps}$ and $-0.180 \mathrm{ps}$ respectively. (e) Numerical simulations and pink curve theoretical predictions.

These experimental observations are confirmed by numerical simulations (Fig. 1(e)) and theoretical developments based on convective instabilities theory [2,3] (pink curve in Fig. (e), derivations details will be provided during the conference).

In conclusion, we showed the impact of synchronization mismatch on MI process. We demonstrated experimentally, numerically, and theoretically that the sideband position depends on the synchronization mismatch, related to the contribution of an odd dispersion term. We expect similar significant effects on broadband OFCs.

\section{References}

[1] E. Obrzud, S. Lecomte, and T. Herr,"Temporal solitons in microresonators driven by optical pulses," Nature Photonics11,600-607 (2017) [2] A. Mussot, E. Louvergneaux, N. Akhmediev, F. Reynaud, L. Delage, and M. Taki, "Optical fiber systems are convectively unstable," Phys. Rev. Lett. 101, (2008).

[3] F. Leo, A. Mussot, P. Kockaert, P. Emplit, M. Haelterman, and M. Taki. "Nonlinear Symmetry Breaking Induced by Third-Order Dispersion in Optical Fiber Cavities". Physical Review Letters 110 (10) (2013), p. 104103 (cit. on pp. 84, 108, 133). 